\title{
MODELAGEM DA BIOMASSA AÉREA EM BRACATINGAIS NATIVOS DA REGIÃO METROPOLITANA DE CURITIBA
}

\author{
Edilson Urbano*, Sebastião do Amaral Machado**, Afonso Figueiredo Filho***, \\ Henrique Soares Koehler**** \\ *Eng. Florestal, M.Sc. - edurbano2@hotmail.com \\ ** Eng. Florestal, Ph.D., Depto. de Ciências Florestais, UFPR, Pesquisador 1A do CNPq - samachado@ufpr.br \\ ***Eng. Florestal, Dr., Depto. de Ciências Florestais, UFPR - afonso.figueiredo@pq.cnpq.br \\ ****Eng. Florestal, Dr., Depto. de Fitotecnia e Fitossanitarismo, UFPR - koehler@ufpr.br \\ Recebido para publicação: 02/08/2007 - Aceito para publicação: 04/09/2007
}

\begin{abstract}
Resumo
A presente pesquisa teve como objetivo desenvolver equações para estimar o peso seco da biomassa por componente (árvore inteira, lenha, galhos grossos (diâmetro $\geq 4 \mathrm{~cm}$ ), galhos finos (diâmetro $\leq 4$ $\mathrm{cm}$ ), folhagem e galhos mortos de bracatinga (Mimosa scabrella Benth.) de povoamentos nativos da Região Metropolitana de Curitiba. Os dados compõem-se de 194 árvores coletadas em bracatingais de diversos municípios. Nessas árvores, foram medidas as variáveis altura total, altura de copa, altura do fuste, diâmetro à altura do peito (DAP) e diâmetro da copa. Cada árvore-amostra foi derrubada e seccionada por componente. O peso verde de cada componente, foi obtido in loco. Amostras de cada componente verde foram coletadas, pesadas e levadas para secagem em estufa até peso seco constante. Posteriormente, foi obtido o peso seco de cada componente por extrapolação dos pesos das amostras. Foram testados 10 modelos aritméticos e 10 logarítmicos, sendo um de cada forma obtido pelo processo de seleção "Forward". O DAP, a altura total e a altura do fuste foram as variáveis independentes mais adequadas para compor as equações estimativas dos pesos secos. Os componentes peso seco do fuste, total e da lenha podem ser estimados satisfatoriamente com as equações desenvolvidas. Já as equações ajustadas para estimar o peso seco das folhas e dos galhos não apresentaram resultados satisfatórios, com erros altos e coeficientes de determinação baixos.

Palavras-chaves: Peso seco; equações; bracatinga.
\end{abstract}

\begin{abstract}
Modeling of above ground biomass for Mimosa scabrella Bentham of native stands from the Curitiba metropolitan region. This research aimed to model the aerial biomass of Mimosa scabrella Bentham of native stands from the Curitiba metropolitan region. The data for this research work came from 190 trees located in several counties of the Curitiba metropolitan region. Total height, crow height, stem height, DBH and crow diameter of every one of the 194 trees were measured. Every sample tree was felled down and sectioned in the following components: stem, thick branches (diameter $\geq 4 \mathrm{~cm}$ ), thin branches (diameter $<4 \mathrm{~cm}$ ), foliage, and dead branches. The green weight of each tree component was weighted in the field. Small sub samples of each tree green component were also weighted in the field and brought to the laboratory for oven dry until unchanged weight. Thus it was obtained the dry weight of the whole component of the 194 trees by extrapolation of the sample weights. DBH, total height and stem height were the independent variables more adequate for estimating dry weights for the components firewood, stem and total. The adjusted equations to estimate dry weight of the leaves and of the branches did not present satisfactory results, with high standard errors and low coefficients of determination $\left(\mathrm{Raj}^{2}\right)$.

Keywords: Dry weight; equations; Mimosa Scabrella.
\end{abstract}

\section{INTRODUÇ̃̃O}

Mimosa scabrella Benth., conhecida popularmente como bracatinga, é uma espécie pioneira, característica das áreas de ocorrência de Araucaria angustifolia, que se desenvolve bem a céu aberto, com crescimento rápido e resistente a geadas. Essa espécie é conhecida pelo seu potencial energético, sendo 
usada principalmente como lenha e carvão. A maior parte da lenha de bracatinga na Região Metropolitana de Curitiba é utilizada pelas indústrias processadoras de cal e/ou calcário, padarias e outras.

Trata-se de uma árvore perenifólia, normalmente com 10 a $18 \mathrm{~m}$ de altura e 20 a $30 \mathrm{~cm}$ de DAP, podendo atingir até $29 \mathrm{~m}$ de altura e $50 \mathrm{~cm}$ de DAP. O tronco pode ser alto e esbelto em maciços, ou curto e ramificado em árvores isoladas. O fuste se desenvolve até $15 \mathrm{~m}$ de comprimento e o diâmetro da copa pode variar de 1,5 m, em povoamentos, até $10 \mathrm{~m}$ em árvores isoladas (ROTTA; OLIVEIRA, 1981). Alguns povoamentos implantados por mudas têm alcançado produtividade de até $36 \mathrm{~m}^{3}$.ha ${ }^{-1}$ ano ou 55 esteres ha- ${ }^{-1}$.ano com casca, sob regeneração artificial (AHRENS, 1981; CARVALHO, 1994).

Grandes superfícies da área metropolitana de Curitiba e outras, principalmente nos estados do Paraná e Santa Catarina, estão florestadas com bracatinga, formando densas associações, em que cerca de $60 \%$ correspondem à bracatinga e o restante engloba mais de 80 espécies (BAGGIO, 1994). Vistos de cima, os bracatingais parecem constituir agrupamentos puros, uma vez que o dossel é exclusivamente formado pelas copas da bracatinga.

A determinação da biomassa está diretamente ligada a questões que envolvem o manejo florestal, uma vez que as informações obtidas no inventário florestal são a base de pesquisas relacionadas ao uso dos recursos naturais, e as decisões sobre o uso eficiente da terra. Em relação ao clima, a biomassa é utilizada para estimar os estoques de carbono, que, por sua vez, são utilizados para estimar a quantidade de $\mathrm{CO}_{2}$ que é liberada, ou pela respiração vegetal, ou por queimadas, ou ainda por decomposição da biomassa.

Segundo Caldeira (2003), o termo biomassa representa a matéria orgânica armazenada em um determinado ecossistema, pois especifica o valor numérico dos componentes presentes, além de ser fundamental nos estudos de ciclagem de nutrientes, conversão de energia, absorção e armazenamento de energia solar. Também possibilita que se tirem conclusões para uma exploração racional dos ecossistemas.

A biomassa de uma árvore expressa em peso pode ser determinada diretamente, por meio da determinação do peso verde de cada componente, ou, por uma amostra representativa dos componentes, estimar-se o teor de umidade e calcular os respectivos pesos secos de cada componente, correlacionando esses valores de biomassa de cada componente da árvore com variáveis de fácil obtenção, como DAP e altura. Dessa forma, obtém-se a estimativa indireta da biomassa seca de uma árvore (SOARES; HOSOKAWA, 1984).

Segundo Pardé (1980), expressar a biomassa em matéria seca é vantajoso na aplicação em determinados mercados madeireiros, caso haja necessidade de explicar a produtividade biológica dos ecossistemas, e pela facilidade em comparações e cálculos.

Para Teixeira (2003), a quantificação da biomassa florestal pode ser feita por dois métodos: o método direto, no qual há a determinação do peso da biomassa fresca e da biomassa seca, e o método indireto, que estima a biomassa por meio de modelos matemáticos a partir de dados de inventários florestais, fazendo a relação entre variáveis como o volume da madeira, o DAP, a altura comercial do tronco, o diâmetro da copa e a altura total das árvores.

Sanquetta; Balbinot (2004) afirmaram que métodos diretos implicam determinações, enquanto métodos indiretos geram estimativas. Assim, determinações não são possíveis em grandes extensões florestais, cabendo a áreas pequenas e amostras tomadas na população para ajustar e calibrar os modelos empregados nas estimativas de biomassa.

Segundo Husch et al. (1982), o aumento do uso de medições de peso para produtos florestais gerou a necessidade de se estimar o peso da madeira em árvores em pé. No início dos anos 60, iniciou-se estudos para estimar peso da porção comercial de árvores em pé e o peso da árvore toda.

A biomassa está relacionada com certas características da árvore, como o diâmetro a 1,30 m e a altura da árvore. Diversos modelos de regressão têm sido empregados na sua estimativa, utilizando essas duas variáveis independentes. A expressão da massa seca como variável dependente é desejável, pois serve de base comparativa dentro de uma espécie e entre espécies, sendo que a massa verde varia com o teor de umidade da madeira (CAMPOS et al., 1992).

Pinheiro (1980), trabalhando com Pinus caribea var. hondurensis e Pinus oocarpa na região de Sacramento $(\mathrm{MG})$, testou 15 variáveis independentes para estimar o peso dos diversos componentes das copas, concluindo ser o DAP a variável simples mais eficiente para todas as estimativas em ambas as espécies. Já Rosot (1980), estudando Pinus taeda, verificou que as variáveis que apresentavam maior 
correlação simples com a variável peso de madeira seca, em ordem decrescente, foram o DAP com casca, a altura comercial e a altura total.

Scolforo et al. (1993) testaram vários modelos tradicionais e outros modelos obtidos pelo método de seleção "stepwise" para estimativa da biomassa do fuste e total de 179 plantas do cerrado, usando como variáveis o CAP, a altura do fuste e a altura total. Dentre os modelos testados, os que tiveram melhor desempenho foram os obtidos pelo método "stepwise", sendo que os modelos tradicionais não tiveram bom desempenho.

Silva (1996), trabalhando com Eucalyptus grandis, ajustou alguns modelos para estimar a biomassa da casca, do tronco, dos galhos, das folhas e do alburno, obtendo coeficientes de determinação variando de 0,93 para as folhas a 0,99 para o tronco aos três anos de idade, com erro padrão da estimativa variando de $26,93 \%$ para as folhas a $4,50 \%$ para a casca aos sete anos de idade.

Wendling (1998) ajustou modelos para estimativa de biomassa para Euterpe edulis. utilizando o DAP e o $D^{2} \mathrm{H}$ como variáveis independentes mais correlacionadas com o peso verde das palmeiras, bem como com o creme comestível do palmito.

Rochadelli (2001), trabalhando com bracatinga, ajustou vários modelos para estimativa de biomassa. Observou o autor que os modelos ajustados apresentaram elevados coeficientes de determinação $\left(\mathrm{R}^{2}\right)$, variando entre 0,98 e 0,99 , com erro padrão da estimativa em porcentagem $\left(\mathrm{S}_{\mathrm{yx}} \%\right)$ variando entre 8,25 e 12,56 .

Nesta pesquisa, teve-se como objetivo desenvolver equações para estimar o peso seco da biomassa para a árvore toda, para a porção lenha, para o fuste, para os galhos grossos, para os galhos finos e para a folhagem.

\section{MATERIAL E MÉTODOS}

\section{Localização e caracterização da área de estudo}

Os dados utilizados para o desenvolvimento desta pesquisa são provenientes de bracatingais nativos da Região Metropolitana de Curitiba, coletados principalmente na região norte, onde sua ocorrência é mais concentrada. Segundo o sistema da classificação de Köppen, o clima da região é do tipo $\mathrm{Cfb}$, que corresponde ao clima temperado, mesotérmico úmido sem estação seca definida, com quatro estações bem definidas, com verões quentes e chuvosos e invernos frios com períodos secos eventuais. A temperatura média do mês mais frio é inferior a $18{ }^{\circ} \mathrm{C}$, e a do mês mais quente fica abaixo de $22^{\circ} \mathrm{C}$. A temperatura média anual se apresenta em torno de $17{ }^{\circ} \mathrm{C}$, com mínimas de $12{ }^{\circ} \mathrm{C}$ e máximas de $23{ }^{\circ} \mathrm{C}$. A precipitação média anual situa-se entre 1.300 e $2.500 \mathrm{~mm}$, com leve diminuição no inverno, não ocorrendo déficits hídricos (MAACK, 1981; IAPAR, 1994).

A região de estudo apresenta relevo predominantemente ondulado a fortemente ondulado. Os solos, na maioria, são Cambissolos álicos, com diferentes graus de profundidade e fertilidade, e, em regra, solos pobres, pouco desenvolvidos, ácidos, com teores elevados de alumínio e baixa saturação de bases.

\section{Obtenção dos dados}

Os dados para o ajuste das funções para estimar a biomassa da bracatinga vieram de uma amostra de 194 árvores cobrindo idades de 4 a 17 anos e classes de diâmetros variando de 4 a $35 \mathrm{~cm}$, provenientes de diversas localidades dos municípios da Região Metropolitana de Curitiba. Para obtenção da biomassa seca de cada componente, procedeu-se da forma relatada a seguir.

No campo, foram escolhidas árvores representativas por classe de idade e por classe de diâmetro. Mediu-se o diâmetro de copa em posições ortogonais, bem como o DAP de cada árvore. Derrubou-se a árvore, mediu-se a altura total, a altura do fuste e a altura de copa. Seccionou-se cada árvore nos componentes fuste, galhos maiores do que $4 \mathrm{~cm}$ de diâmetro, galhos menores do que $4 \mathrm{~cm}$ de diâmetro e folhagem. Pesou-se cada componente da árvore, recolheram-se amostras de cada componente em sacos plásticos e posteriormente pesaram-se todas essas amostras verdes.

No laboratório, as amostras foram fracionadas em tamanhos menores e posteriormente secas em estufa a $103^{\circ} \mathrm{C}$ até peso constante. Pesaram-se as amostras secas e calculou-se o percentual da biomassa seca de cada componente.

Os pesos secos dos componentes de cada árvore foram obtidos por simples consideração do percentual do peso seco sobre o peso verde, obtido após a secagem das amostras. O peso seco total foi obtido somando-se os pesos de todos os componentes, e o peso seco da lenha foi obtido pela soma do 
peso do componente fuste com o peso dos galhos até o diâmetro mínimo de $4 \mathrm{~cm}$. Os teores percentuais de biomassa das amostras de cada componente foram calculados com base na relação: Teor de matéria seca $=($ Peso seco $/$ Peso verde $) * 100$.

\section{Equações para estimar o peso seco da biomassa}

Vários modelos matemáticos existentes na literatura pertinente foram testados. Para estimativa do peso seco da biomassa dos componentes das árvores de bracatinga, ajustaram-se 20 modelos matemáticos por componente, sendo 10 aritméticos e 10 logarítmicos, tendo como variável dependente o peso seco dos componentes (ps em $\mathrm{kg}$ ) e como variáveis independentes o diâmetro à altura do peito (DAP) $(\mathrm{d}$ em $\mathrm{cm}$ ), a altura total (h em m), a altura do fuste (hf em $\mathrm{m}$ ), a altura de copa (hc em $\mathrm{m}$ ), o diâmetro de copa (dc em $\mathrm{m}$ ) e a combinação dessas variáveis na forma aritmética e na logarítmica. Em cada uma das formas, uma equação foi obtida pelo método de seleção "forward" para cada um dos componentes das árvores analisadas. Os modelos utilizados são apresentados na tabela 1.

Tabela 1. Modelos utilizados no ajuste das equações estimativas do peso seco de cada componente analisado nas árvores de bracatinga.

Table 1. Used models for fitting dry weight equations for each tree component.

\begin{tabular}{ccc}
\hline $\mathbf{N}^{\mathbf{0}}$ & Aritmética & Logarítmica \\
\hline 1 & $\mathrm{p}_{\mathrm{s}}=\mathrm{b}_{0}+\mathrm{b}_{1} \mathrm{~d}$ & $\ln _{\mathrm{s}}=\mathrm{b}_{0}+\mathrm{b}_{1} \operatorname{lnd}$ \\
2 & $\mathrm{p}_{\mathrm{s}}=\mathrm{b}_{0}+\mathrm{b}_{1} \mathrm{~d}^{2}$ & $\ln \mathrm{p}_{\mathrm{s}}=\mathrm{b}_{0}+\mathrm{b}_{1} \operatorname{lnd} \mathrm{d}^{2}$ \\
3 & $\mathrm{p}_{\mathrm{s}}=\mathrm{b}_{0}+\mathrm{b}_{1} \mathrm{~d}^{3}$ & $\ln \mathrm{p}_{\mathrm{s}}=\mathrm{b}_{0}+\mathrm{b}_{1} \operatorname{lnd} 3$ \\
4 & $\mathrm{p}_{\mathrm{s}}=\mathrm{b}_{0}+\mathrm{b}_{1} \mathrm{~d}+\mathrm{b}_{2} \mathrm{~h}$ & $\ln \mathrm{p}_{\mathrm{s}}=\mathrm{b}_{0}+\mathrm{b}_{1} \operatorname{lnd}+\mathrm{b}_{2} \operatorname{lnh}$ \\
5 & $\mathrm{p}_{\mathrm{s}}=\mathrm{b}_{0}+\mathrm{b}_{1} \mathrm{~d}+\mathrm{b}_{2} \mathrm{hf}$ & $\ln \mathrm{p}_{\mathrm{s}}=\mathrm{b}_{0}+\mathrm{b}_{1} \operatorname{lnd}+\mathrm{b}_{2} \operatorname{lnhf}$ \\
6 & $\mathrm{p}_{\mathrm{s}}=\mathrm{b}_{0}+\mathrm{b}_{1} \mathrm{~d}^{2} \mathrm{~h}$ & $\ln \mathrm{p}_{\mathrm{s}}=\mathrm{b}_{0}+\mathrm{b}_{1} \operatorname{lnd} \mathrm{l}^{2} \mathrm{~h}$ \\
7 & $\mathrm{p}_{\mathrm{s}}=\mathrm{b}_{0}+\mathrm{b}_{1} \mathrm{~d}^{2} \mathrm{hf}$ & $\operatorname{lnp}_{\mathrm{s}}=\mathrm{b}_{0}+\mathrm{b}_{1} \operatorname{lnd} \mathrm{hf}^{2} \mathrm{hf}$ \\
8 & $\mathrm{p}_{\mathrm{s}}=\mathrm{b}_{0}+\mathrm{b}_{1} \mathrm{dc}$ & $\operatorname{lnp}_{\mathrm{s}}=\mathrm{b}_{0}+\mathrm{b}_{1} \operatorname{lndc}$ \\
9 & $\mathrm{p}_{\mathrm{s}}=\mathrm{b}_{0}+\mathrm{b}_{1} \mathrm{~d}^{2}+\mathrm{b}^{2} \mathrm{dc}$ & $\ln \mathrm{p}_{\mathrm{s}}=\mathrm{b}_{0}+\mathrm{b}_{1} \operatorname{lnd} \mathrm{f}^{2}+\mathrm{b}_{2} \operatorname{lndc}$ \\
10 & Forwarder & Forwarder \\
\hline
\end{tabular}

A seleção do melhor modelo foi baseada nos seguintes critérios de escolha: coeficiente de determinação ajustado $\left(\mathrm{R}^{2}{ }_{\mathrm{aj}}\right)$, erro padrão da estimativa em porcentagem $(\mathrm{Syx} \%)$ e análise gráfica dos resíduos em porcentagem.

Os modelos logarítmicos foram corrigidos quanto à discrepância logarítmica pelo Fator de Correção de Meyer antes do recálculo do Syx para a unidade quilograma.

\section{RESULTADOS E DISCUSSÃO}

Na tabela 2, são apresentadas as estatísticas das variáveis medidas em campo e dos pesos secos obtidos por componente das árvores de bracatinga com idades entre 4 e 17 anos. Observa-se que os pesos dos componentes e das variáveis medidas no campo apresentam valores altos de coeficiente de variação, ocasionado pela grande variabilidade de forma e tamanho das árvores nos povoamentos nativos.

Os teores percentuais de matéria seca encontrados em cada componente das árvores analisadas, com os valores máximos, médios e mínimos, bem como com o coeficiente de variação de cada um são apresentados na tabela 3. Com base nesses teores, calculou-se o peso seco de biomassa para cada componente e, somando-se esses pesos, obtiveram-se o peso total e o peso da porção lenha das árvores.

\section{Peso seco total}

De uma forma geral, as equações desenvolvidas para estimar o peso seco total apresentaram $\mathrm{R}^{2} \mathrm{e}$ Syx variando de 0,67 a 0,97 e de 18,12 a 58,30\%, respectivamente. A equação de melhor performance foi obtida pelo método de seleção "forward" na forma aritmética $\left(\mathrm{R}^{2}=0,97\right.$ e Syx\% $\left.=18,12\right)$, com uma distribuição residual sem tendências (Figura 1), conforme segue: $\mathrm{ps}=276,1587-33,5871 d+1,19453 d^{2}+0,07883 d^{2} h-0,3085 d h+6,32376 d c-690,7668 d^{-1}-0,45352 h c-$
$0,000628 d^{3} h f-0,001852 d^{3} h$ 
Tabela 2. Estatísticas das variáveis medidas em campo e dos pesos secos por componente.

Table 2. Statistics of measured variables and dry weight by tree component.

\begin{tabular}{lcccccccccrr}
\hline \multirow{2}{*}{ Estatísticas } & \multicolumn{4}{c}{ Variáveis medidas no campo } & \multicolumn{6}{c}{ Peso seco dos componentes } \\
\cline { 2 - 12 } & \multicolumn{1}{c}{$\mathbf{d}$} & $\mathbf{d}_{\mathbf{c}}$ & \multicolumn{1}{c}{$\mathbf{h}$} & \multicolumn{1}{c}{$\mathbf{h}_{\mathbf{f}}$} & \multicolumn{1}{c}{$\mathbf{h}_{\mathbf{c}}$} & Fuste & $\mathbf{G} \geq \mathbf{4}$ & $\mathbf{G}<\mathbf{4}$ & Folhas & Total & Lenha \\
\hline Mínimo & 4,30 & 0,55 & 9,15 & 2,56 & 1,70 & 2,12 & 0,40 & 0,74 & 0,09 & 4,76 & 2,12 \\
Média & 13,10 & 3,01 & 13,91 & 8,74 & 5,17 & 61,22 & 29,40 & 6,01 & 4,95 & 95,52 & 81,08 \\
Mediana & 11,50 & 2,50 & 13,50 & 8,70 & 4,95 & 35,00 & 7,87 & 3,86 & 3,11 & 48,35 & 40,58 \\
Máximo & 35,00 & 8,85 & 21,80 & 15,45 & 12,00 & 261,36 & 221,02 & 50,09 & 47,03 & 586,90 & 481,57 \\
CV & 49,46 & 58,25 & 20,29 & 29,06 & 38,30 & 103,13 & 164,70 & 104,12 & 115,12 & 113,28 & 116,95 \\
n & 194 & 194 & 194 & 194 & 194 & 194 & 152 & 194 & 194 & 194 & 194 \\
\hline
\end{tabular}

Tabela 3. Estatísticas do teor de matéria seca em \% dos componentes das árvores.

Table 3. Statistics of dry weigh contend in percent of the tree components.

\begin{tabular}{lccccc}
\hline \multirow{2}{*}{ Estatísticas } & \multicolumn{5}{c}{ Componentes } \\
\cline { 2 - 6 } & Fuste & Casca & Galhos $\geq \mathbf{4}$ & Galhos <4 & Folhas \\
\hline Mínimo & 38,68 & 34,14 & 30,84 & 40,00 & 30,48 \\
Médio & 48,49 & 45,23 & 49,37 & 50,87 & 39,87 \\
Máximo & 58,79 & 57,03 & 59,48 & 56,83 & 53,76 \\
Coeficiente de variação & 7,74 & 11,46 & 7,76 & 6,03 & 10,50 \\
\hline
\end{tabular}
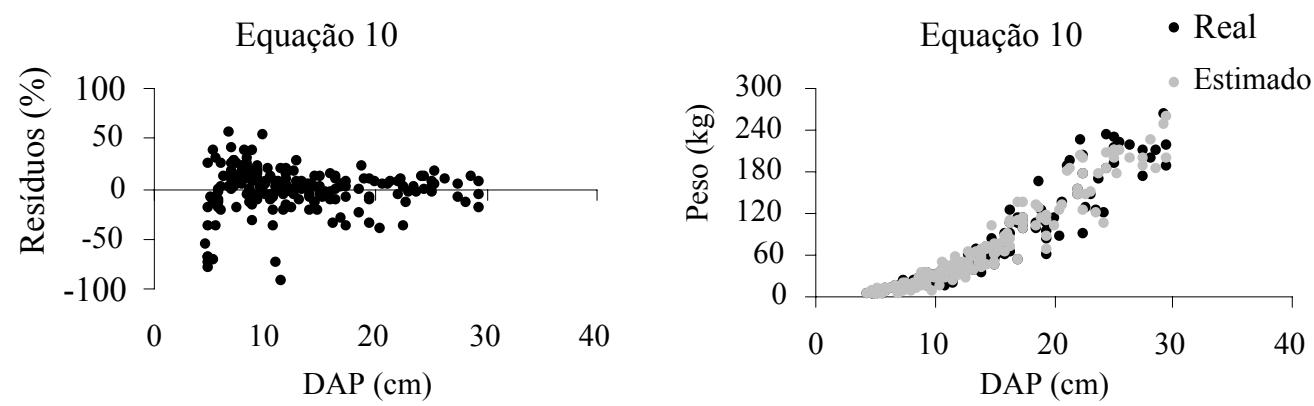

Figura 1. Resíduos em porcentagem e distribuição do estimado sobre o real para a equação de melhor estimativa do peso seco total.

Figure 1. Residual distribution and estimated over observed values of the best equation for estimating total dry weight.

Dentre as equações ajustadas apenas com a variável DAP, a de melhor desempenho inclui o DAP na sua forma quadrática, com $\mathrm{R}^{2}$ e Syx de 0,96 e 21,68\%, respectivamente. Essa equação apresenta uma boa distribuição de resíduos (Figura 2), tendo a seguinte forma: ps $=-11,83006+0,50917 d^{2}$. Essa é a equação de uso mais prático dentre todas.

Soares; Hosokawa (1984) também trabalharam no desenvolvimento de equações para estimar a biomassa da bracatinga. Para a biomassa total, obtiveram, para uma equação logarítmica, cuja variável foi apenas o DAP, $\mathrm{R}^{2}$ de 0,92 . Essa mesma equação, na presente pesquisa, apresentou $\mathrm{R}^{2}$ de 0,88 e Syx $\%$ de 35,48 . Esses autores também ajustaram modelos cujas variáveis independentes foram o DAP e a altura total, resultando numa equação logarítmica com $\mathrm{R}^{2}$ de 0,93 , enquanto que neste trabalho a mesma equação obteve $\mathrm{R}^{2}$ de 0,93 e Syx de $27,04 \%$. A melhor dentre todas as equações ajustadas para o peso seco total por Soares; Hosokawa (1984) apresentou $\mathrm{R}^{2}=0,94$. Baggio (1994) encontrou como melhor modelo para o peso seco total uma equação com $\mathrm{R}^{2}$ de 0,98 e Syx\% de 18,8. Portanto, os resultados obtidos nesta pesquisa são similares aos encontrados por esses autores. 
Equação 2

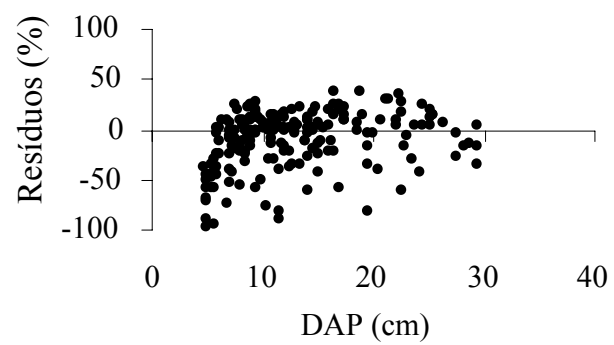

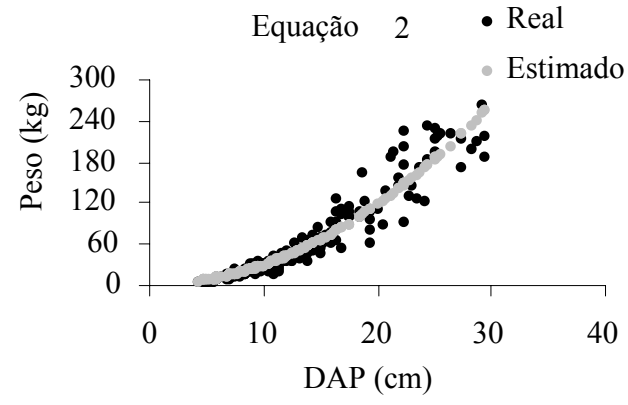

Figura 2. Resíduos em porcentagem e distribuição do estimado sobre o real para a equação recomendada para a estimativa do peso seco total.

Figure 2. Residual distribution and estimated over observed values of the recommended equation for total dry weight estimate.

\section{Peso seco da lenha}

As equações ajustadas para estimar o peso seco da lenha apresentaram erro padrão da estimativa variando de $23,47 \%$ até $62,81 \%$, e coeficiente de determinação variando de 0,71 a 0,96 . A melhor equação para esse componente foi a aritmética, obtida pelo método de seleção "forward", tendo, como variáveis independentes no modelo, a combinação do DAP com a altura total, da seguinte forma: ps = $6,669413+0,209204 d^{2}+0,012728 d^{2} h$. Essa é a equação indicada para uso, por ser a mais precisa e apresentar variáveis de fácil obtenção.

A distribuição de resíduos dessa equação (Figura 3) mostra que há uma tendência de subestimativa ao longo da linha de regressão das classes de menores diâmetros, porém boa distribuição ao longo das outras classes de DAP.

Equação 10

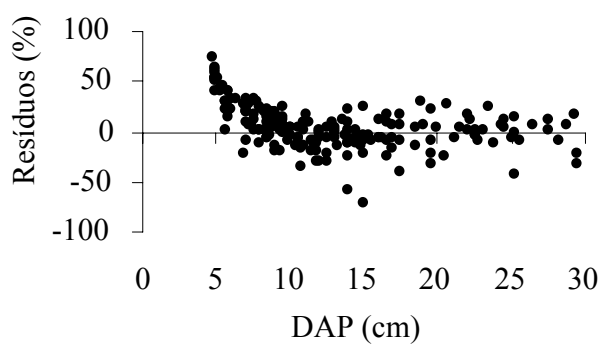

Equação $10 \quad$ Real

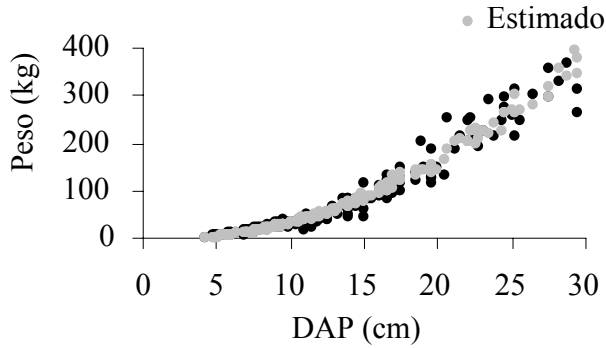

Figura 3. Resíduos em porcentagem e distribuição do estimado sobre o real da equação de melhor ajuste para estimativa do peso seco da lenha.

Figure 3. Residual distribution and estimated over observed values of the best equation for estimating fire wood dry weight.

Baggio (1994) ajustou um modelo para lenha cujo resultado foi bastante semelhante ao melhor modelo encontrado neste trabalho, com $\mathrm{R}^{2}$ de 0,98 e Syx de 18,6\%. A variável independente do modelo de Baggio foi apenas o DAP. A base de dados desse autor foi restrita à idade de sete anos, portanto, com uma distribuição diamétrica mais homogênea e uma variação menor do peso seco, resultante da concentração dos dados numa única idade.

Silva (1996) destacou a alta correlação que o DAP apresenta com o peso dos componentes das árvores, atingindo um coeficiente de determinação $\left(\mathrm{R}^{2}\right)$ maior do que 0,95 e distribuição de resíduos aceitáveis para os modelos ajustados. Afirmou também que, devido à menor correlação do DAP com os 
pesos dos galhos e das folhas, faz-se necessário o seu uso na forma quadrática e associado com a altura total, para composição do modelo matemático.

\section{Peso seco do fuste}

As equações desenvolvidas para estimar o peso seco do fuste apresentaram $\mathrm{R}^{2}$ e Syx variando de 0,66 a 0,97 e de 17,57 a $58,98 \%$, respectivamente. A equação de melhor desempenho foi obtida pelo método de seleção "forward", na forma aritmética $\left(\mathrm{R}^{2}=0,97\right.$ e Syx\% $\left.=17,57\right)$, com uma distribuição residual sem tendências (Figura 4), conforme segue: ps $=-44,90105+0,0123 \mathrm{~d}^{2} \mathrm{hf}+0,3002 \mathrm{dh}+4,2165 \mathrm{dc}$ $+197,8859 * \mathrm{~d}^{-1}-1,7854 \mathrm{hc}$.
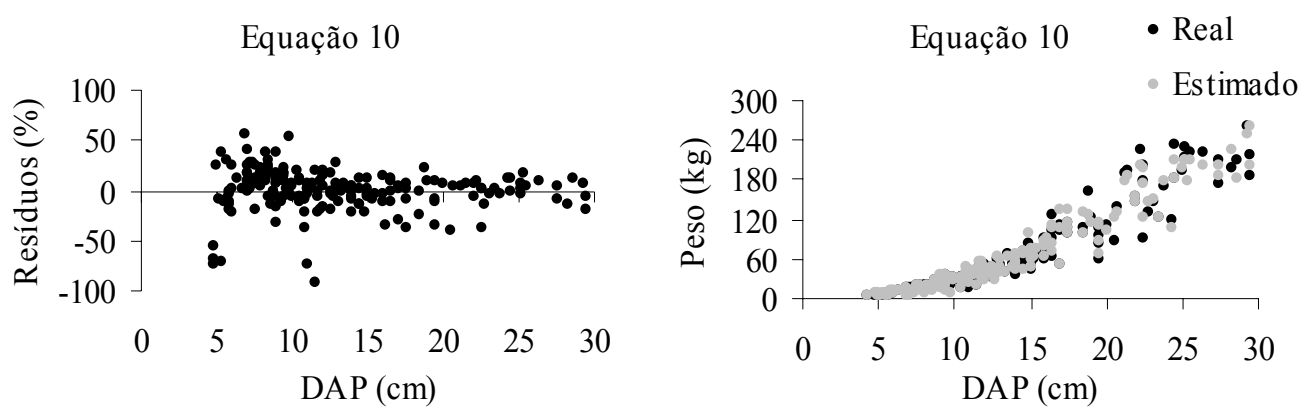

Figura 4. Resíduos e comportamento dos dados estimados sobre os dados reais da equação de melhor ajuste para estimativa do peso seco do fuste.

Figure 4. Residual distribution and estimated over observed values of the best equation for estimating stem dry weight.

A equação recomendada para estimativa do peso seco do fuste é $\ln p s=-2,88197+2,00026 \operatorname{lnd}+$ 0,72683lnhf, com um erro padrão da estimativa (Syx\%) de 20,10\% e coeficiente de determinação $\left(\mathrm{R}^{2}\right)$ de 0,96, com distribuição residual sem tendência ao longo da linha de regressão (Figura 5). Para essa equação, o fator de correção da discrepância logarítmica de Meyer é 1,0109. Essa equação tem a vantagem de não usar o diâmetro de copa e a altura de copa na sua composição, porém gera estimativas similares à equação de melhor ajuste.

Equação 5

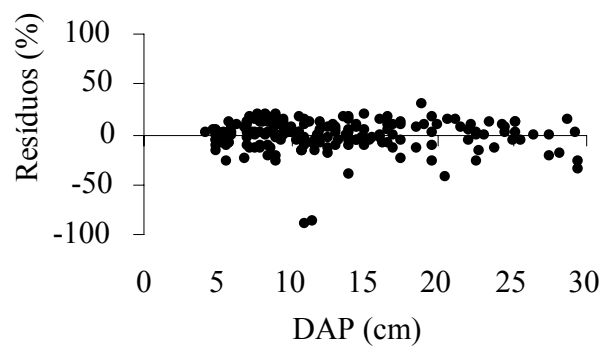

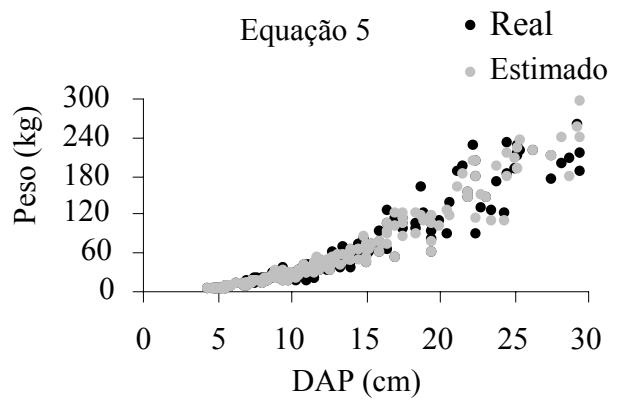

Figura 5. Resíduos e comportamento dos dados estimados sobre os dados reais da equação recomendada para estimativa do peso seco do fuste.

Figure 5. Residual distribution and estimated over observed values of the recommended equation for stem dry weight estimate.

\section{Peso seco dos galhos maiores do que $4 \mathrm{~cm}$ de diâmetro}

O desempenho de todas as 20 equações foi insatisfatório, com erro padrão da estimativa variando de 43,16 a $105,7 \%$, e o coeficiente de determinação variando de 0,45 a 0,91 . O menor erro e o maior coeficiente de determinação foram os da equação obtida pelo método de seleção "forward", que usa como 
variáveis independentes o DAP, a altura do fuste, a altura total e o diâmetro de copa em suas combinações, compondo a equação: ps $=0,56781+0,03782 \mathrm{~d}^{2}+0,02497 \mathrm{~d}^{2} \mathrm{~h}-0,02907 \mathrm{~d} 2 \mathrm{hf}-0,06395 \mathrm{dh}$. Recomenda-se o uso dessa equação para estimativa do peso seco dos galhos maiores ou iguais a $4 \mathrm{~cm}$ de diâmetro, por ser a mais precisa dentre todas as ajustadas.
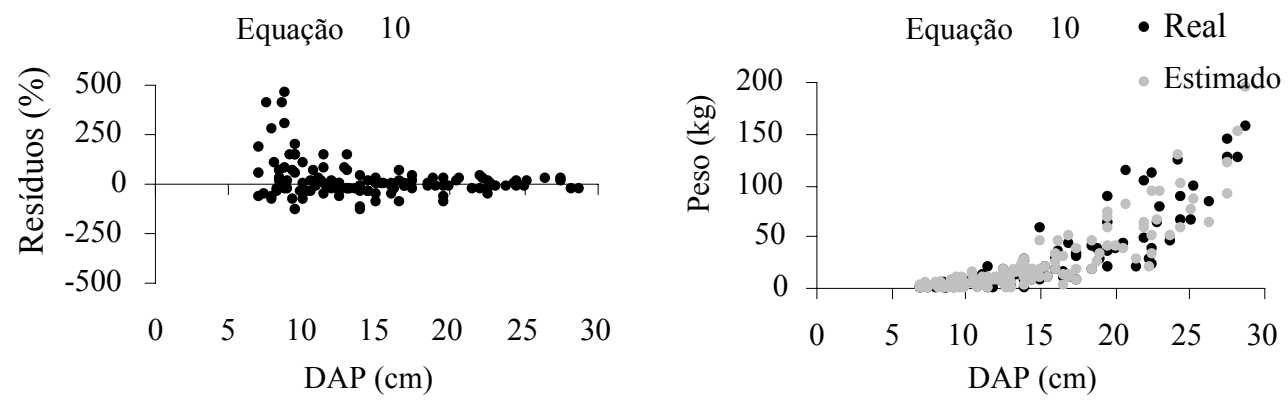

Figura 6. Resíduos e comportamento dos dados estimados sobre os dados reais da equação estimativa do peso seco dos galhos $\geq 4 \mathrm{~cm}$ de diâmetro.

Figure 6. Residual distribution and estimated over observed values of the recommended equation of branch dry weight with diameter $\geq 4 \mathrm{~cm}$.

Observando-se a figura 6, verifica-se que a distribuição dos resíduos apresentam alta dispersão, com tendência a subestimativa nas classes de menor diâmetro e sem tendência para diâmetros maiores. $\mathrm{O}$ comportamento dos dados estimados sobre os dados reais mostra que, apesar do erro alto, a equação, na média, explica o peso seco encontrado para as árvores de bracatinga, podendo ser usada com reserva para grandes quantidades de dados, pois dessa forma os erros serão compensados.

\section{Peso seco dos galhos menores do que $4 \mathrm{~cm}$ de diâmetro}

As equações apresentaram erro padrão da estimativa variando de 36,01 a 67,55\% e coeficiente de determinação variando de 0,59 a 0,85. O menor erro e o maior coeficiente de determinação foram os da equação obtida pelo método "forward", resultando a equação ps $=24,94795-4,15905 d+0,27256 d^{2}-$ $0,00473 \mathrm{~d}^{3}+0,55408 \mathrm{dc}-44,67591 \mathrm{~d}^{-1}$, que usa como variável independente o DAP (d), em suas combinações, e o diâmetro de copa $(\mathrm{dc})$.

Analisando os gráficos da figura 7, observa-se que os resíduos apresentaram-se não-tendenciosos e a curva dos valores estimados sobre os dados reais mostra que a equação, em média, estima bem os pesos secos dos galhos menores do que $4 \mathrm{~cm}$ de diâmetro.

Equação 10

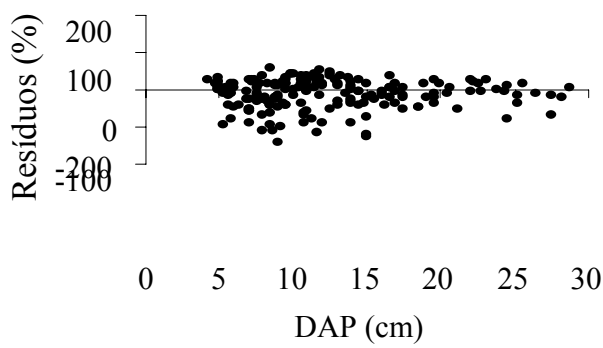

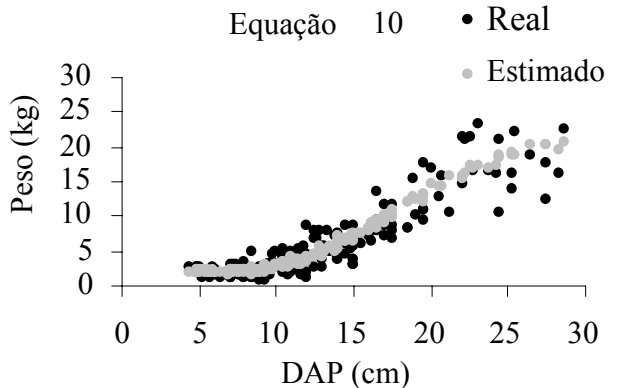

DAP $(\mathrm{cm})$

Figura 7. Resíduos e comportamento dos dados estimados sobre os dados reais da equação de melhor ajuste para estimativa do peso seco dos galhos $\leq 4 \mathrm{~cm}$ de diâmetro.

Figure 7. Residual distribution and estimated over observed values of the best equation for estimating branch dry weight with diameter $\leq 4 \mathrm{~cm}$. 
Resultados similares foram encontrados por Baggio (1994). Sua equação de melhor ajuste para os ramos (galhos $<3 \mathrm{~cm}$ de diâmetro) apresentou um $\mathrm{R}^{2}$ de 0,89 e Syx de $45,1 \%$.

$\mathrm{O}$ uso da equação de melhor desempenho é dificultado pela complexidade de obtenção da variável diâmetro de copa em campo, podendo ser substituída por uma equação que usa como variáveis independentes o DAP e a altura do fuste em sua composição e apresentou $\mathrm{R}^{2}$ de 0,81 e Syx de $40,74 \%$. Essa equação tem a seguinte forma: $p s=-2,81521+0,92477 \mathrm{~d}-0,33001 \mathrm{hf}$.
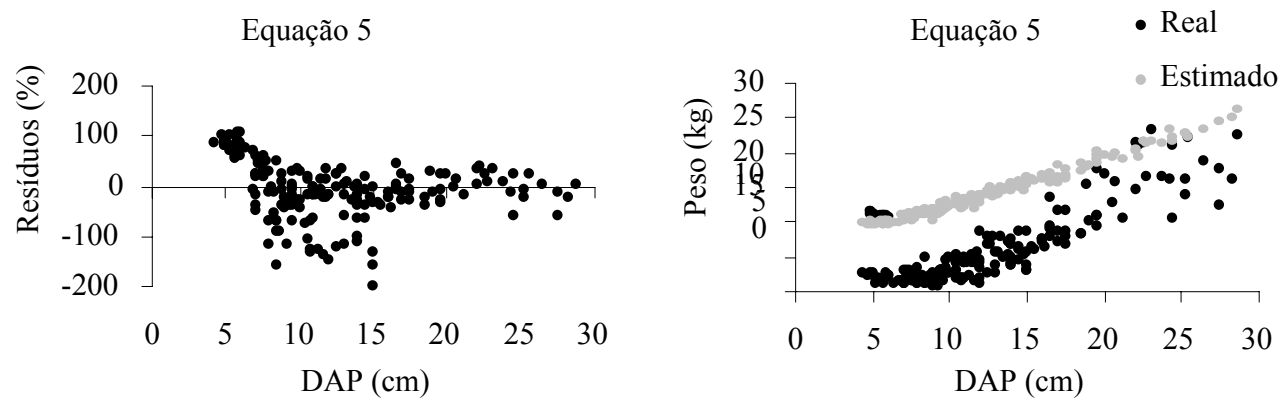

Figura 8. Resíduos e comportamento dos dados estimados sobre os dados reais da equação recomendada para estimativa do peso seco dos galhos menores do que $4 \mathrm{~cm}$ de diâmetro.

Figure 8. Residual distribution and estimated over observed values of the recommended equation of branch dry weight with diameter $\leq 4 \mathrm{~cm}$.

Observando os gráficos da figura 8, observa-se que os resíduos apresentaram-se com uma pequena tendência de subestimativa nas classes de menores diâmetros. Já a curva dos valores estimados sobre os dados reais mostra que a equação tem uma tendência linear ao longo da distribuição dos dados reais, mas que, em média, estima bem os pesos secos dos galhos menores do que $4 \mathrm{~cm}$ de diâmetro.

\section{Peso seco das folhas}

As 20 equações ajustadas para estimar o peso seco das folhas tiveram erro padrão da estimativa variando de 36,67 a $71,90 \%$, e coeficiente de determinação variando de 0,62 a 0,87 . O menor erro e o maior coeficiente de determinação foram da equação aritmética ps $=-6,50888+0,73822 \mathrm{~d}-0,17025 \mathrm{~h}+$ $0,76127 \mathrm{dc}+20,50980 \mathrm{~d}^{-1}$, ajustada através do método de seleção "forward". O gráfico de resíduos apresentado na figura 9 mostra que não há tendência ao longo da linha de regressão e que os valores estimados sobre os valores reais têm boa distribuição.
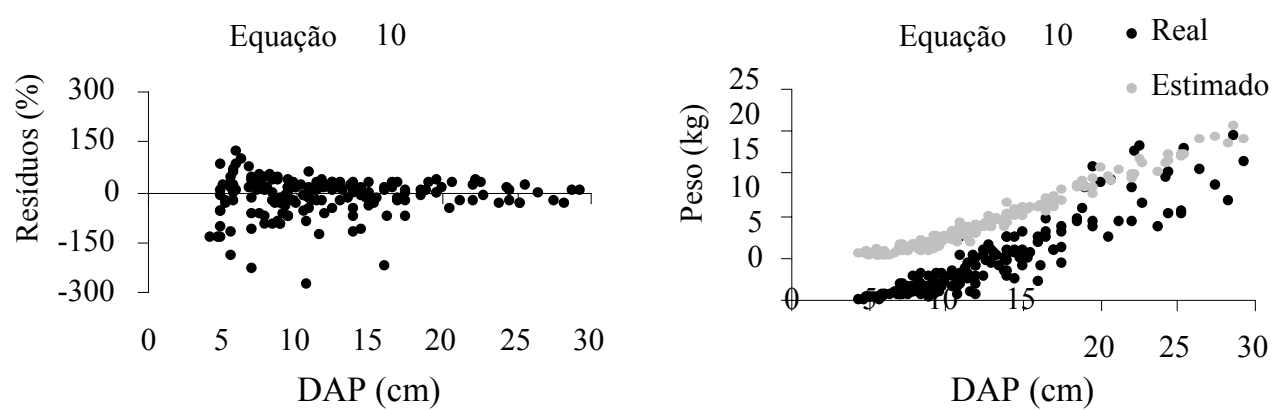

Figura 9. Resíduos e comportamento dos dados estimados sobre os dados reais da equação de melhor ajuste para estimativa do peso seco das folhas.

Figure 9. Residual distribution and estimated over observed values of the best equation for estimating leaves dry weight. 
Baggio (1994) obteve como melhor ajuste para a biomassa verde (folhas) uma equação com $\mathrm{R}^{2}$ de 0,91 e um Syx de 40,9\% para povoamentos em idade de corte ( 7 anos). Na época, esse mesmo autor sugeriu que fossem ajustados modelos com as variáveis diâmetro de copa e altura de copa para se obterem equações com melhor desempenho. Porém, neste trabalho, o uso dessas variáveis na composição dos modelos não revelarou nenhuma melhora no desempenho das equações, mesmo usando-se uma base de dados com maior diversidade de idades e aplicando-se o método de seleção de variáveis "forward". Brown (1965), usando dados coletados em Pinus resinosa e Pinus banksiana, concluiu que o DAP é a melhor variável simples para estimar o peso da copa das árvores, e que a combinação do DAP mais o comprimento da copa multiplicado pelo DAP aumentou a acuracidade das estimativas.

Como a equação de melhor desempenho é também a de maior complexidade, alternativamente pode-se usar a equação que tem como variáveis independentes o DAP e a altura do fuste em suas combinações, e que apresenta um $\mathrm{R}^{2}$ de 0,85 e um Syx de 38,88\%, formando a seguinte equação: ps = $2,12406+0,81507 \mathrm{~d}-0,25382 \mathrm{~h}$.

Como pode ser observado na figura 10, os resíduos apresentam-se tendenciosos, com subestimativa nas classes de menores diâmetros e com alta amplitude nos seus valores. Os dados estimados sobre os dados reais apresentam uma distribuição linear, mostrando que a equação ajustada não foi flexível o suficiente para se ajustar à distribuição dos pesos da folhagem.
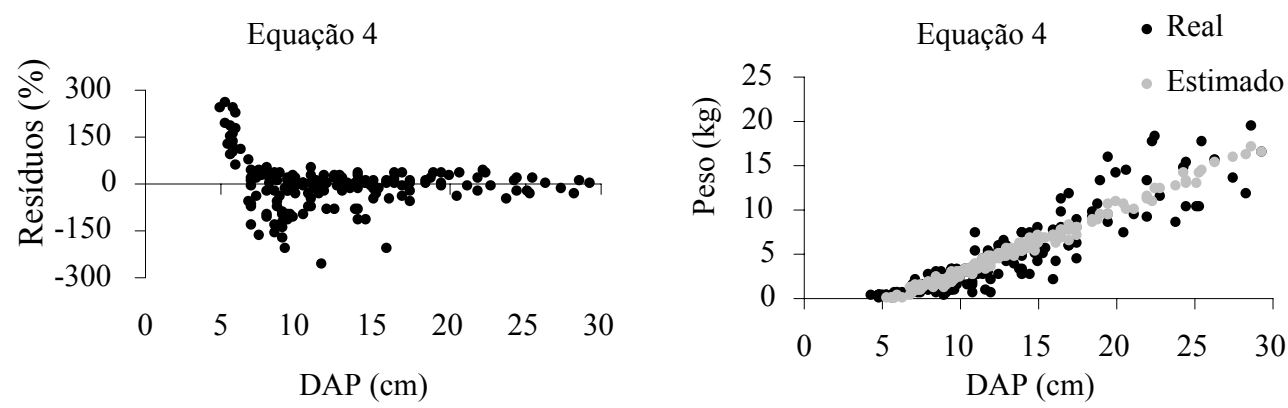

Figura 10. Resíduos e comportamento dos dados estimados sobre os dados reais da equação alternativa para estimativa do peso seco das folhas.

Figure 10. Residual distribution and estimated over observed values of the recommended equation for leaves dry weight estimate.

\section{CONCLUSÕES}

A inclusão das variáveis altura de copa e diâmetro de copa não trouxe melhorias aos modelos e, portanto parece não ser necessárias, principalmente pelas dificuldades operacionais para se obtê-las.

O DAP, a altura do fuste e a altura total foram as variáveis mais adequadas para explicar a variabilidade do peso seco da biomassa dos componentes da bracatinga.

Entre todos os componentes da biomassa estudados, o peso seco do fuste foi o componente que melhor pode ser estimado com modelos de regressão, seguido pelo peso seco total e pelo peso seco da lenha.

As equações geradas para estimar o peso seco dos galhos e da folhagem, muito embora tenham ajustes aceitáveis, apresentaram elevados erros, o que mostra a dificuldade de estimar esses componentes por meio de modelos de regressão.

As equações aqui ajustadas permitem a quantificação indireta da biomassa dos povoamentos de bracatinga da Região Metropolitana de Curitiba, sendo importantes na qualificação do estoque de madeira usada como fonte energética. Quando utilizadas conjuntamente com equações estimativas de carbono, podem fornecer o estoque de carbono nos povoamentos, permitindo assim que a bracatinga venha a fazer parte do mercado de créditos de carbono, proporcionando, no futuro, mais uma fonte de renda para os pequenos proprietários rurais. 


\section{REFERÊNCIAS}

AHRENS, S. Um modelo matemático para volumetria comercial de bracatinga (Mimosa scabrella Benth.). In: SEMINÁRIO SOBRE ATUALIDADES E PERSPECTIVAS FLORESTAIS. Bracatinga uma alternativa para reflorestamento, Curitiba 4., 1981, Curitiba. Anais... Curitiba: EMBRAPA-URPFCS, 1981. p. 77-90. (Documentos URPFCS, n. 5).

BAGGIO, A. J. Estudio sobre el sistema agroforestal tradicional de la bracatinga (Mimosa scabrella Benth.) en Brasil: productividad, manejo de residuos y elaboracion de compost. 242 p. Tese (Doutorado) - Universidad Politécnica de Madrid, Madrid, 1994.

BROWN, J. K. Estimating crown fuel weights of red pine and jack pine. Washington, DC: U. S. Forest Service, 1965. 12 p. (Research Paper LS, n. 20).

CALDEIRA, M. V. W. Determinação de biomassa e nutrientes em uma Floresta Ombrófila Mista Montana em General Carneiro, Paraná. 176 f. Tese (Doutorado em Engenharia Florestal) - Setor de Ciências Agrárias, Universidade Federal do Paraná, Curitiba, 2003.

CAMPOS, J. C. C.; SILVA, J. A. da; VITAL, B. R. Volume e biomassa do tronco e da copa de Eucalipto de grande porte. Revista Árvore, Viçosa, MG, v. 16, n. 3, p. 319-336, 1992.

CARVALHO, P. E. R. Espécies florestais brasileiras: recomendações silviculturais, potencialidades e uso da madeira. Colombo: EMBRAPA-CNPF, 1994. 640 p.

DRAPER, N. R.; SMITH, H. Applied Regression Analysis. $2^{\text {nd }}$ ed. New York, J. Wiley \& Sons, 1980. $709 \mathrm{p}$.

HUSCH, B.; MILLER, C. I.; BEERS, T. W. Forest Mensuration. $3^{\text {rd }}$ ed. New York: J. Wiley \& Sons, 1982. $401 \mathrm{p}$.

IAPAR - Instituto Agronômico do Paraná. Cartas climáticas do estado do Paraná: 1994. Londrina, 1994.

MAACK, R. Geografia física do Estado do Paraná. 2. ed. Rio de Janeiro: J. Olympio; Curitiba: Secretaria da Cultura e do Esporte do Governo do Estado do Paraná. 1981. 450 p.

PARDÉ, J. Forest Biomass. Forestry Abstracts, v. 41, n. 8, p. 343-362, 1980.

PINHEIRO, G. S. Estimativa de pesos de copas de Pinus caribea var. hondurensis e Pinus oocarpa através de parâmetros dendrométricos. 105 f. Dissertação (Mestrado em Engenharia Florestal) - Setor de Ciências Agrárias, Universidade Federal do Paraná, Curitiba, 1980.

ROCHADELLI, R A estrutura de fixação dos átomos de carbono em reflorestamento (Estudo de caso: Mimosa scabrella Bentham, Bracatinga). $86 \mathrm{f}$. Tese (Doutorado em Engenharia Florestal) Setor de Ciências Agrárias, Universidade Federal do Paraná, Curitiba, 2001.

ROSOT, N. C. Estimativa do peso de madeira seca de Pinus taeda L. por ocasião do primeiro desbaste. Dissertação (Mestrado em Engenharia Florestal) - Setor de Ciências Agrárias, Universidade Federal do Paraná, Curitiba, 1980.

ROTTA, E.; OLIVEIRA, Y. M. M. Área de distribuição natural da bracatinga (Mimosa scabrella). In: SEMINÁRIO SOBRE ATUALIDADES E PERSPECTIVAS FLORESTAIS. Bracatinga uma alternativa para reflorestamento, Curitiba 4., 1981, Curitiba. Anais... Curitiba: EMBRAPA-URPFCS, 1981. p. 117122. (Documentos URPFCS, n. 5).

SANQUETTA, C. R; BALBINOT, R. Metodologia para determinação de biomassa florestal. In: SANQUETTA, C. R; BALBINOT, R.; ZILOTTO, M. A. B. (Eds.). Fixação de carbono: atualidades, projetos e pesquisa. Curitiba: UFPR/Laboratório de Inventário Florestal: Instituto Ecoplan: PELD, 2004. p. $77-94$ 
SCOLFORO, J. R. S.; LIMA, J. T.; SILVA, S. T. da. Equações de biomassa e volume para cerrado Senso Stricto. In: CONGRESSO FLORESTAL PANAMERICANO, 1.: CONGRESSO FLORESTAL BRASILEIRO, 7., 1993, Curitiba. Anais... São Paulo: SBS/SBEF. v. 2. p. 508-510.

SILVA, H. D. Modelos matemáticos para a estimativa da biomassa e do conteúdo de nutrientes em plantações de Eucalyptus grandis Hill (ex. Maiden) em diferentes idades. $110 \mathrm{f}$. Tese (Doutorado em Engenharia Florestal) - Setor de Ciências Agrárias, Universidade Federal do Paraná, Curitiba, 1996.

SOARES, R. V.; HOSOKAWA, R. T. Estimativa da biomassa energética de árvores de bracatinga. Brasil florestal, Brasília, DF, n. 8. p. 37 - 48, 1984.

TEIXEIRA, L. M. Influência da Intensidade de exploração seletiva de madeira no crescimento e respiração do tecido lenhoso das árvores em uma Floresta Tropical de terra-firme na região de Manaus. 61 p.. Dissertação (Mestrado) - Universidade Federal do Amazonas, Manaus, 2003.

WENDLING, J. L. G. Modelos matemáticos de crescimento e de produção em biomassa para árvores de Euterpe edulis Mart. plantadas a céu aberto. 168 f. Dissertação (Mestrado em Engenharia Florestal) - Setor de Ciências Agrárias, Universidade Federal do Paraná, Curitiba, 1998. 